\title{
Temperature dependence of alkali-antimonide photocathodes: Evaluation at cryogenic temperatures
}

\author{
M. A. Mamun, ${ }^{1}$ M. R. Hernandez-Flores, ${ }^{2}$ E. Morales, ${ }^{3}$ C. Hernandez-Garcia, ${ }^{1}$ and M. Poelker ${ }^{1}$ \\ ${ }^{1}$ Thomas Jefferson National Accelerator Facility, Newport News, Virginia 23606, USA \\ ${ }^{2}$ Metropolitan Autonomous University, Mexico City, Distrito Federal 02200, Mexico \\ ${ }^{3}$ National Autonomous University of Mexico, Mexico City, Distrito Federal 04510, Mexico
}

(Received 11 July 2017; published 24 October 2017)

\begin{abstract}
$\mathrm{Cs}_{x} \mathrm{~K}_{y} \mathrm{Sb}$ photocathodes were manufactured on a niobium substrate and evaluated over a range of temperatures from 300 to $77 \mathrm{~K}$. Vacuum conditions were identified that minimize surface contamination due to gas adsorption when samples were cooled below room temperature. Measurements of the photocathode spectral response provided a means to evaluate the photocathode band gap dependence on the temperature and to predict the photocathode quantum efficiency at $4 \mathrm{~K}$, a typical temperature at which superconducting radio frequency photoguns operate.
\end{abstract}

DOI: 10.1103/PhysRevAccelBeams.20.103403

\section{INTRODUCTION}

Alkali-antimonide photocathodes have been used since the 1950s for a wide range of applications, including photodetectors and emitters [1], and more recently as electron sources inside photoguns that generate relativistic picosecond-long electron bunches to form high-brightness, high-average current electron beams for accelerator applications such as high-power free electron lasers and energy recovery linacs (ERLs) [2,3]. Alkali-antimonide photocathodes are most often operated at ambient temperature; however, some applications require or benefit from operation at cryogenic temperatures. For example, in neutrino physics experiments the photomultipliers tubes (PMTs) must operate at temperatures below $200 \mathrm{~K}$ while maintaining a high quantum efficiency $(\mathrm{QE}>10 \%)$ and fast response times $(<1 \mathrm{~ns})$ [4]. Similarly, cooling reduces the detector's thermionic dark current for astronomical photometry PMTs [5].

Recent cW accelerator initiatives requiring an unprecedented high-average beam current of the order of $100 \mathrm{~mA}$ for electron coolers [6] and high repetition rate ERLs [7] have focused on utilizing alkali-antimonide photocathodes inside superconducting radio frequency (SRF) photoguns, which effectively combines the extremely high gradient capability of the SRF photogun operated in the cw mode with the proven robustness of this type of photocathode [2,3]. An SRF photogun can be designed to accommodate a photocathode at room temperature [8], but, from a thermal engineering perspective, it would be better to operate the

Published by the American Physical Society under the terms of the Creative Commons Attribution 4.0 International license. Further distribution of this work must maintain attribution to the author(s) and the published article's title, journal citation, and DOI. photocathode at a cryogenic temperature. And cold photocathodes enable compelling physics research, providing reduced intrinsic emittance to meet the transverse coherence length requirements of state-of-the-art ultrafast electron diffraction (UED) and inverse Compton scattering applications [9].

For PMTs, when photocathodes are grown on transparent nonconductive substrates, operation at a cryogenic temperature causes sensitivity loss due to the increased photocathode resistivity [4], but this is not a problem for photocathodes grown on electrically conductive substrates. Any reduction in quantum efficiency $(\mathrm{QE})$ observed when a photocathode is cooled must be a result of adsorbed gas contamination on the photocathode surface and/or the shifting band gap that results from mechanical stress on the photocathode crystal structure. Brookhaven National Laboratory researchers reported a drop in QE of about 20\% when a $\mathrm{K}_{2} \mathrm{CsSb}$ photocathode was cooled to $166 \mathrm{~K}$ inside their $704 \mathrm{MHz}$ SRF gun [10], while Cornell researchers reported a drop of $\sim 80 \%$ in $\mathrm{QE}$ at $680 \mathrm{~nm}$ when a $\mathrm{Cs}_{3} \mathrm{Sb}$ was cooled to $90 \mathrm{~K}$ inside a $20 \mathrm{kV}$ dc gun [9].

In this work, we describe the successful fabrication of $\mathrm{Cs}_{x} \mathrm{~K}_{y} \mathrm{Sb}$ photocathodes on a niobium substrate. The photocathodes demonstrated reasonably high QE at room temperature in the range of $5 \%-13 \%$ at $532 \mathrm{~nm}$. These values are comparable to $\mathrm{Cs}_{x} \mathrm{~K}_{y} \mathrm{Sb}$ photocathodes grown on other substrates $[11,12]$. When samples were cooled to 196 and $77 \mathrm{~K}$, the QE values decreased due to the expected modification of the semiconductor band gap [13]. Photocathode QE also decreased when samples were cooled as a result of surface contamination caused by gas adsorption [10], but this ill effect could be minimized by reducing the water partial pressure inside the vacuum apparatus. In contrast to the referenced work mentioned above, the photocathode QE at $532 \mathrm{~nm}$ decreased by only $\sim 50 \%$ when the photocathode was cooled from room 
temperature to $77 \mathrm{~K}$. By measuring the photocathode QE spectral response over a wide range of temperatures, the band gap dependence on the temperature was empirically determined and extrapolated to $4 \mathrm{~K}$, which is the nominal liquid helium operating temperature of L-band SRF photoguns [14].

\section{EXPERIMENTAL DETAILS}

Photocathodes were manufactured inside the vacuum apparatus described in Ref. [11]. A niobium substrate $(15 \times 15 \times 1 \mathrm{~mm})$ cut from an ingot of large-grain niobium was polished with $1 \mu \mathrm{m}$ diamond grit to achieve a surface roughness of $\sim 10 \mathrm{~nm} \mathrm{rms}[15,16]$. The niobium substrate was attached to the end of a sample holder composed of a hollow stainless steel tube with a thin stainless steel and molybdenum end plate using a thin tantalum retaining ring. The sample holder was designed to accommodate a heater but also served as a cryostat when the heater was removed. For these measurements, the sample holder was filled with ice water, dry ice (frozen $\mathrm{CO}_{2}$ ), or liquid nitrogen $\left(\mathrm{LN}_{2}\right)$. The substrate remained cold for many hours with just one filling. A thermal analysis of the sample holder indicated that the substrate temperature was nearly identical to the temperature of the ice water, frozen $\mathrm{CO}_{2}$, or $\mathrm{LN}_{2}$.

To make a photocathode, the niobium substrate was heated to $600{ }^{\circ} \mathrm{C}$ for $4 \mathrm{~h}$ to rid the surface of adsorbed gas and then allowed to cool. Antimony was then deposited onto the substrate at $200{ }^{\circ} \mathrm{C}$, followed by codeposition of alkali species at $120^{\circ} \mathrm{C}$. A number of photocathodes were manufactured. For subsequent reactivation of photocathodes, the old photocathode was heated to $200^{\circ} \mathrm{C}$ for $2 \mathrm{~h}$ to dissociate and desorb the retained alkali. Heat cleaning at a higher temperature was avoided, because the $\mathrm{Sb}$ was observed to diffuse into the $\mathrm{Nb}$ substrate. This approach resulted in increasing $\mathrm{Sb}$ thickness for repeated photocathode activations. For the first fabrication, $\mathrm{Sb}$ pellets were heated inside a sapphire crucible by passing an electrical current through a tungsten heater for $25 \mathrm{~min}$. For later reactivations, the $\mathrm{Sb}$ evaporation duration was reduced to $10 \mathrm{~min}$. The partial pressure of $\mathrm{Sb}$ was $6.0( \pm 0.5) \times 10^{-8} \mathrm{~Pa}$ as measured using a residual gas analyzer (RGA). (Note that the total pressure reported by the RGA compared reasonably well to the total pressure of the system as inferred by the ion pump current reading. Although absolute pressure values are reported here, the RGA served primarily as a tool to measure relative pressure changes.) The antimony thickness for the studied activations ranged from $\sim 0.3$ to $1.0 \mu \mathrm{m}$, based on calibration studies performed previously [11]. During alkali deposition, the photocathode was biased at $-284 \mathrm{~V}$ and illuminated with a low-power green laser $(4 \mathrm{~mW}, 532 \mathrm{~nm})$. Alkali as a mixture of Cs and $\mathrm{K}$ was evaporated from an effusion source, and the alkali deposition continued until the photocurrent reached a maximum value. The details of the activation process are available in Ref. [11]. Typical photocathode fabrication took about $4-6 \mathrm{~h}$, including the initial substrate heating and chemical deposition.

A vacuum was achieved using nonevaporable getter pumps (two GP100 MK5 flange-mounted pumps and two WP950 NEG modules from SAES ${ }^{\circledR}$ Getters, with a hydrogen pumping speed of 480 and $2300 \mathrm{Ls}^{-1}$, respectively) and an ion pump (45 S Titan ion ${ }^{\mathrm{TM}}$ pump from Gamma vacuum with a nitrogen pumping speed of $\sim 40 \mathrm{Ls}^{-1}$ ). A turbo-molecular pump was used to pump down the apparatus, to achieve a vacuum level suitable to energize the ion pump. Two vacuum conditions were studied, baked and unbaked, with the key difference being the amount of residual water vapor inside the chamber.

\section{RESULTS AND DICUSSIONS}

Initially, a photocathode was fabricated in the vacuum chamber that had been vented with clean dry nitrogen but not baked. Under these conditions, the total pressure was $\sim 1.8 \times 10^{-7} \mathrm{~Pa}$, and the partial pressure of $\mathrm{H}_{2} \mathrm{O}$ was $\sim 1.7 \times 10^{-8} \mathrm{~Pa}$. The photocathode $\mathrm{QE}$ at $532 \mathrm{~nm}$ was evaluated while the sample was repeatedly cooled to $77 \mathrm{~K}$ and allowed to return to room temperature. Photocathode $\mathrm{QE}$ at $532 \mathrm{~nm}$ was expected to decrease at cold temperatures because of the temperature-induced band gap increase and due to a reduction in occupied defect states above the valence band level $[1,13]$, but, upon letting the sample warm to room temperature, the $\mathrm{QE}$ did not return to the original value, indicating that the photocathode surface had become contaminated by adsorbed gas while cold (Fig. 1). Photocathode QE could be fully restored by briefly heating the sample to $\sim 185^{\circ}\left( \pm 15^{\circ}\right) \mathrm{C}$ which served to desorb the contamination, thereby restoring a clean surface condition. The QE increase was accompanied by a transient rise in $\mathrm{H}_{2} \mathrm{O}$ partial pressure, supporting the speculation that water vapor was the source of the contamination. Notice in Fig. 1 that, after brief heating and QE recovery, the behavior could be reproduced, with a QE reduction occurring each time the sample was cooled to the liquid nitrogen temperature, and $\mathrm{QE}$ restoration achieved by sample heating.

The vacuum chamber was baked at $180{ }^{\circ} \mathrm{C}$ for $72 \mathrm{~h}$, and another $\mathrm{Cs}_{x} \mathrm{~K}_{y} \mathrm{Sb}$ photocathode was grown on the original niobium substrate. For baked vacuum conditions, the total pressure was $\sim 2.2 \times 10^{-8} \mathrm{~Pa}$, and the partial pressure of $\mathrm{H}_{2} \mathrm{O}$ was $\sim 9.3 \times 10^{-10} \mathrm{~Pa}$. Under these improved vacuum conditions, with each cooldown from room temperature, the photocathode QE at $532 \mathrm{~nm}$ was consistently between $6.2 \%$ and $7.5 \%$ (see Fig. 2). Photocathode QE at room temperature increased from $7 \%$ to $13 \%$ during the first three cryogenic cycles, suggesting an abundance of alkali upon initial fabrication and the gradual optimization of the photocathode stoichiometry over time [17].

A comparison of vacuum chamber partial pressures, unbaked versus baked conditions, highlighted the enhanced 




FIG. 1. Photocathode QE at $532 \mathrm{~nm}$ following successive iterations of sample cooling and heating inside the unbaked vacuum chamber. Note that QE did not restore following a return to room temperature, indicating that the vacuum conditions promoted surface contamination via gas adsorption. The blue text boxes indicate the temperature at which the sample was heated to rejuvenate the $\mathrm{QE}$ and the percentage increase in $\mathrm{H}_{2} \mathrm{O}$ partial pressure relative to the system total pressure during the brief heating period, as reported by the RGA.

presence of chemically reactive species $\mathrm{H}_{2} \mathrm{O}$ in the unbaked vacuum chamber, but other gas species, $\mathrm{H}_{2}, \mathrm{CH}_{4}, \mathrm{CO}_{2}$, and Ar, were present at enhanced levels, too, and these gases might also play a role in $\mathrm{QE}$ reduction at cryogenic temperatures [10].

The photocathode that was fabricated in the baked vacuum chamber was studied further using a wavelength tunable white-light supercontinuum laser [18] to evaluate the photocathode QE spectral response from 425 to $825 \mathrm{~nm}$ at three temperatures: 275,195 , and $77 \mathrm{~K}$. The experimental spectral response data were fitted according to a model developed by Fowler [19]:

$$
\ln \left(\frac{Q E}{T^{2}}\right)=B+\ln \left[f\left(\frac{h \nu-V_{o}}{\kappa_{B} T}\right)\right]=B+\ln [f(x)]
$$

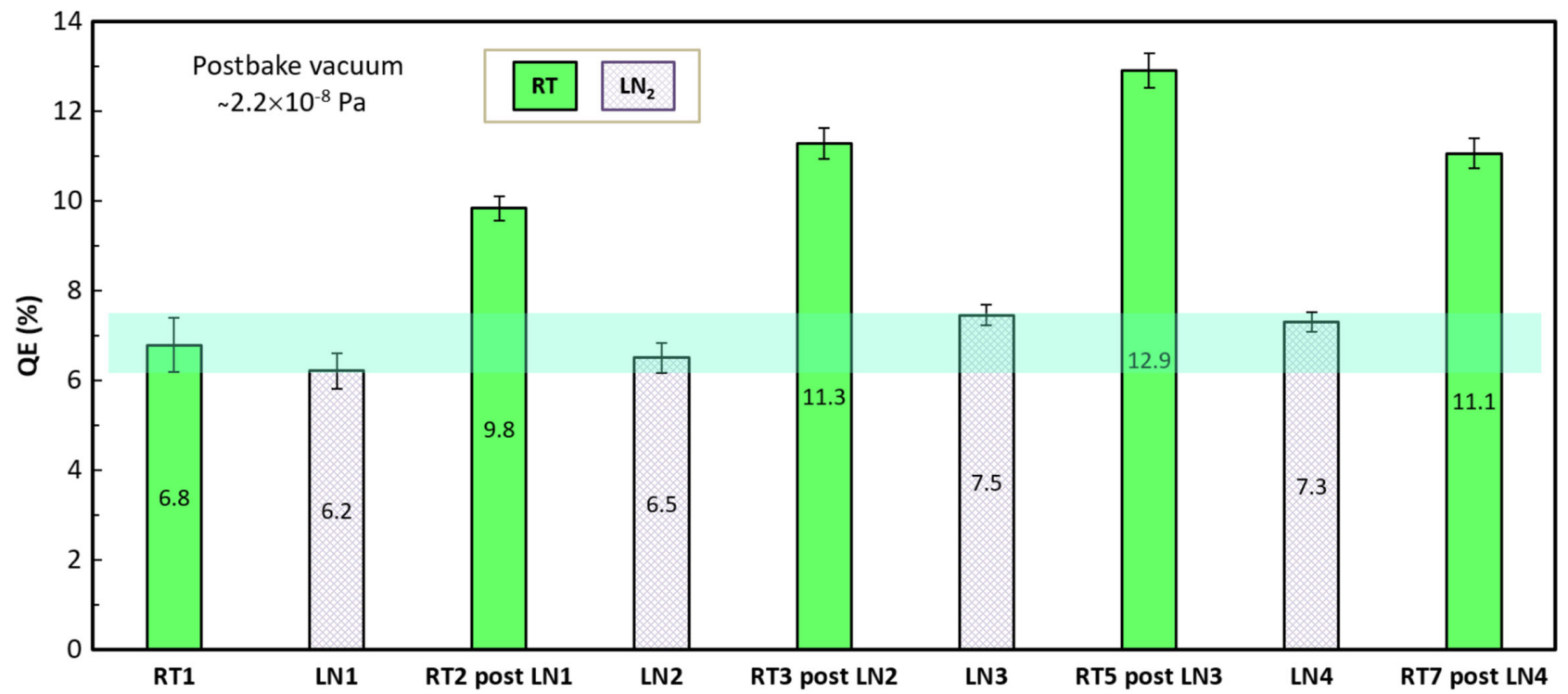

FIG. 2. Photocathode QE at $532 \mathrm{~nm}$ following successive iterations of sample cooling and heating inside the baked vacuum chamber. Photocathode QE at $77 \mathrm{~K}$ was consistently $\sim 6 \%$ to $7 \%$, and when the sample was returned to room temperature, photocathode QE actually increased from $\sim 7 \%$ to $13 \%$. 

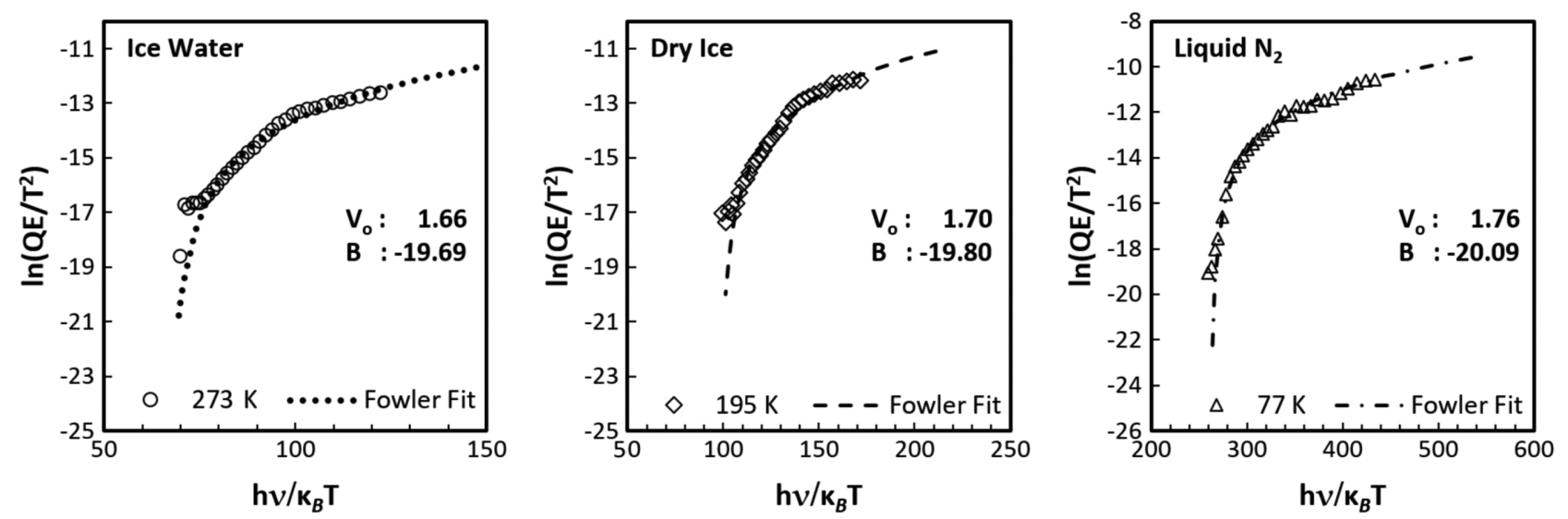

FIG. 3. QE spectral response from the $\mathrm{Cs}_{x} \mathrm{~K}_{y} \mathrm{Sb}$ photocathode grown on the Nb substrate measured at the indicated temperatures using a wavelength tunable laser. The axes units were chosen based on Fowler's model and Eq. (1).

where $V_{o}=E_{g}+E_{A}$ is the photoemission threshold (photoelectric work function) and $B$ is a term related to the material's electron density of states, the optical reflectivity, and electron transport to the surface and is indirectly related to the initial $\mathrm{QE}$ at $\mathrm{RT}$ immediately prior to cryogenic measurement. The term $E_{g}$ is the band gap energy, and $E_{A}$ is the electron affinity. For $x=\left(\frac{h \nu-V_{o}}{\kappa_{B} T}\right)$, the term $f(x)$ can be expanded using the following series approximation:

$$
\begin{aligned}
f(x) & =e^{x}-\frac{e^{2 x}}{4}+\frac{e^{3 x}}{9}-\cdots \quad \text { for } x \leq 0, \\
& =\frac{\pi^{2}}{6}+\frac{x^{2}}{2}-\left(e^{-x}-\frac{e^{-2 x}}{4}+\frac{e^{-3 x}}{9}-\cdots\right) \quad \text { for } x \geq 0 .
\end{aligned}
$$

Figure 3 shows the QE spectral response measurements with fits based on Fowler's model for various temperatures.

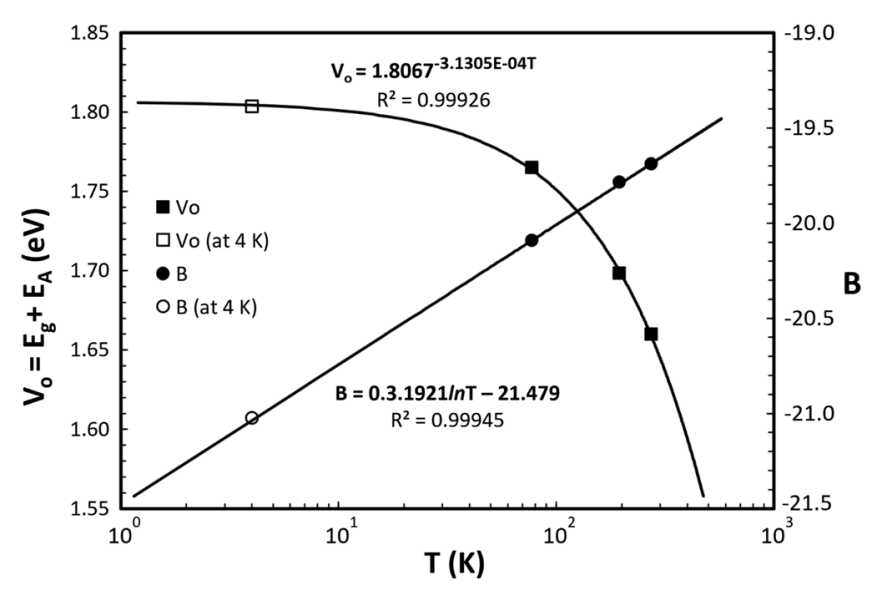

FIG. 4. Exponential fit of the photoemission threshold $V_{o}$ (square) and parameter $B$ (circle), each obtained from the $\mathrm{QE}$ spectral fit for each temperature indicated in Fig. 3. The open markers indicate extrapolation of $V_{o}$ and $B$ values to $4 \mathrm{~K}$.
Using these fits, the photoemission threshold $V_{o}$ and parameter $B$ were determined for each temperature, allowing the determination of the temperature-dependent functions $V_{o}(T)$ and $B(T)$ as shown in Fig. 4.

From the fits shown in Fig. 4, the photoemission threshold $V_{o}$ and parameter $B$ were evaluated at $4 \mathrm{~K}$, which is a typical operating temperature of an SRF photogun. Figure 5 shows the spectral response curves of the $\mathrm{Cs}_{x} \mathrm{~K}_{y} \mathrm{Sb}$ photocathode evaluated at 273, 195, and $77 \mathrm{~K}$ together with Fowler model fits and the estimated spectral response at $4 \mathrm{~K}$ (solid line). The model-based estimated QE of the $\mathrm{Cs}_{x} \mathrm{~K}_{y} \mathrm{Sb}$ photocathode grown on a $\mathrm{Nb}$ substrate at $4 \mathrm{~K}$ and $532 \mathrm{~nm}$ is about $1.4 \%$, assuming the initial $\mathrm{QE}$ of $11.5 \%$ at room temperature.

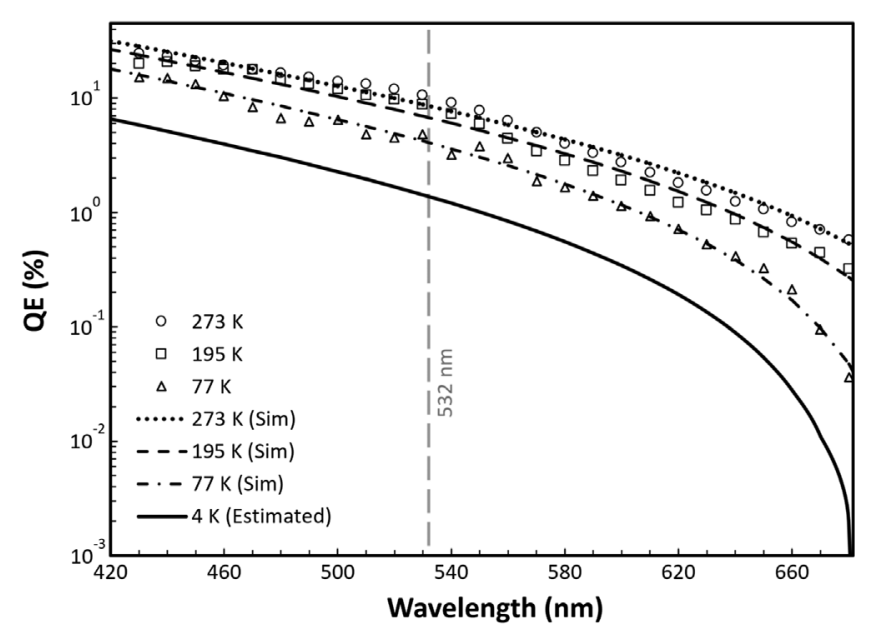

FIG. 5. Measured QE spectral response for the $\mathrm{Cs}_{x} \mathrm{~K}_{y} \mathrm{Sb}$ photocathode grown on the $\mathrm{Nb}$ substrate for the indicated temperatures: circles, ice water; rectangle, dry ice; triangles, liquid nitrogen. The dashed lines through QE data points correspond to fits based on Fowler's model (see the text). The solid black line is a simulated spectral response using the photoemission threshold $V_{o}$ and fit parameter $B$ extrapolated to $4 \mathrm{~K}$. The vertical dashed line at $532 \mathrm{~nm}$ indicates a $\mathrm{QE}$ of $1.4 \%$ at $4 \mathrm{~K}$. 
It must be noted that Fowler's model is based on the Fermi free electron model, which is a good approximation for metal photocathodes, because it assumes the photoemission threshold $V_{o}$ to be independent of the temperature. From the plots above, this is obviously not true of the $\mathrm{Cs}_{x} \mathrm{~K}_{y} \mathrm{Sb}$ semiconductor photocathode. So, from a photocathode physics perspective, our analysis of the spectral response data is not explicitly correct, but rather it represents an expedient practical means to extrapolate $\mathrm{QE}$ data to $4 \mathrm{~K}$.

\section{DISCUSSION}

The temperature dependence of $\mathrm{Cs}_{x} \mathrm{~K}_{y} \mathrm{Sb}$ photocathodes grown on a niobium substrate was evaluated, providing benchmark QE values for low emittance beam applications such as UED and to suggest possible use at a cryogenic temperature inside SRF photoguns, which are considered an ideal choice for accelerator applications that require continuous wave beams at high-average current and bunch charge [20,7]. Under vacuum conditions that minimize the presence of water vapor, the observed QE decrease associated with operation at cold temperatures was inferred to be predominantly related to mechanical stress and the associated shifting of the band gap energy. Our experimental results span a relatively broad range of temperatures, from 300 to $77 \mathrm{~K}$, allowing fitting of the data using the photoemission model of Fowler [19]. The extrapolation of measurements to $4 \mathrm{~K}$ suggests that $\mathrm{Cs}_{x} \mathrm{~K}_{y} \mathrm{Sb}$ photocathodes grown on $\mathrm{Nb}$ substrates could provide $\mathrm{QE} \sim 1.4 \%$ at $532 \mathrm{~nm}$, which is sufficiently high to support the production of highaverage current, milliampere beams using typical commercial lasers. Our extrapolation assumes that the electrical resistivity of the $\mathrm{Cs}_{x} \mathrm{~K}_{y} \mathrm{Sb}$ photocathode does not increase at $4 \mathrm{~K}[4,13]$. It is possible that successful photocathode operation at $4 \mathrm{~K}$ could simplify SRF photogun design, for example, by reducing the cryogenic burden on the liquid helium source and by minimizing the complexity of the rf choke joint that is difficult to design when the photocathode and surrounding surfaces are at different temperatures.

\section{ACKNOWLEDGMENTS}

Authored by Jefferson Science Associates, LLC under U.S. DOE Contract No. DE-AC05-06OR23177. The U.S. Government retains a non-exclusive, paid-up, irrevocable, world-wide license to publish or reproduce this manuscript for U.S. Government purposes. We are grateful to one of the referees for useful comments on the Fowler model.

[1] W. E. Spicer, Photoemissive, Photoconductive, and Optical Absorption Studies of Alkali-Antimony Compounds, Phys. Rev. 112, 114 (1958).

[2] D. Dowell, K. Davis, K. Friddell, E. Tyson, C. Lancaster, L. Milliman, R. Rodenburg, T. Aas, M. Bemes, S. Betherl, P. Johnson, K. Murphy, C. Whelen, J. Adamski, D.
Pistoresi, D. Shoffstall, G. Busch, and Remelius, in Proceedings of the 15th Particle Accelerator Conference, PAC-1993, Washington, DC, 1993 (IEEE, New York, 1993), pp. 2967-2969.

[3] B. Dunham, J. Barley, A. Bartnik, I. Bazarov, L. Cultrera, J. Dobbins, G. Hoffstaetter, B. Johnosn, R. Kaplan, S. Karkare, V. Kostroun, Y. Li, M. Liepe, X. Liu, F. Loehl, J. Maxson, P. Quigley, J. Reilly, D. Rice, D. Sabol, E. Smith, K. Smolenski, M. Tigner, V. Vesherevich, D. Widger, and Z. Zhao, Record high-average current from a high-brightness photoinjector, Appl. Phys. Lett. 102, 034105 (2013).

[4] H. M. Araujo, A. Bewick, D. Davidge, J. Dawson, T. Ferbel, A. S. Howard, W. G. Jones, M. Joshi, V. Lebedenko, I. Liubarsky, J. J. Quenby, T. J. Summer, and F. Nerves, Low-temperature of 35 photomultiplier tubes for the ZEPLIN III experiment, Nucl. Instrum. Methods Phys. Res., Sect. A 521, 407 (2004).

[5] A. T. Young, Temperature effects in photomultpliers and astronomical photometry, Appl. Opt. 2, 51 (1963).

[6] Y. Derbenev and Y. Zhang, Electron cooling for electrons-ion collider at JLab, in Proceedings of COOL 2009, Lanzhou, China (JACoW, 2009), FRM2MCCO01, pp. 181-184.

[7] T. Kamps, W. Anders, A. Frahm, J. Knobloch, O. Kugeler, J. Rudoph, A. Matveenki, A. Neumann, T. Qaust, M. Schenk, M. Schuster, P. Kneisel, J. Sekutowicz, R. Nietubyc, and I. Will, Status and perspectives of superconducting radio-frequency gun development for BERLinPro, J. Phys. Conf. Ser. 298, 012009 (2011).

[8] T. Xin et al., Design of a high-bunch-charge 112-MHz superconducting RF photoemission electron source, Rev. Sci. Instrum. 87, 093303 (2016).

[9] L. Cultrera, S. Karkare, H. Lee, X. Liu, I. Bazarov, and B. Dunham, Cold electron beams from cryocooled, alkali antinomide photocathodes, Phys. Rev. ST Accel. Beams 18, 113401 (2015).

[10] H. Xie, I. Ben-Zvi, T. Rao, T. Xin, and E. Wang, Experimental measurements and theoretical model of the cryogenic performance of bialkali photocathode and characterization with Monte Carlo simulation, Phys. Rev. Accel. Beams 19, 119901 (2016).

[11] M. A. Mamun, A. A. Elmustafa, C. Hernandez-Garcia, R. Mammei, and M. Poelker, Effect of Sb thickness on the performance of bialkali-anitmonide photocathodes, J. Vac. Sci. Technol. A 34, 021509 (2016).

[12] L. Cultrera, I. V. Bazarov, J. V. Conway, B. Dunham, S. Karkare, Y. Li, X. Liu, J. M. Maxson, and K. W. Smolenski, Growth and characterization of bialakli photocathodes for Cornell ERL injector, in Proceedings of the 24th Particle Accelerator Conference, PAC-2011, New York, 2011 (IEEE, New York, 2011), WEP244.

[13] W. E. Spicer and F. Wooten, Photoemission and photomultipliers, Proc. IEEE 51, 1119 (1963).

[14] J. Teichert, H. Büttig, P. Evtushenko, D. Janssen, U. Lehnert, P. Michel, and Ch. Schneider, Status review of superconducting RF photo-injectors, in Proceedings of the 11th Workshop on RF Superconductivity, Lubeck/Travemunder, Germany, 2003 (DESY, Lubeck/Travemunder, 2003), TUO07, pp. 244-248.

[15] N. D. Theodore, B. C. Holloway, D. M. Manos, R. Moore, C. Hernandez, T. Wang, and H. F. Dylla, Nitrogen-implanted 
silicon oxynitride: A coating for suppressing field emission from stainless steel used in high-voltage applications, IEEE Trans. Plasma Sci. 34, 1074 (2006).

[16] M. BastaniNejad, M. A. Mamun, A. A. Elmustafa, P. Adderley, J. Clark, S. Covert, J. Hansknecht, C. Hernandez-Garcia, M. Poelker, R. Mammei, K. SurlesLaw, and P. Williams, Evaluation of niobium as candidate electrode material for dc high voltage photoelectron guns, Phys. Rev. ST Accel. Beams 15, 083502 (2012).

[17] D. H. Dowell, S. Z. Bethel, and K. D. Friddell, Results from the average power laser experiment photocathode injector test, Nucl. Instrum. Methods Phys. Res., Sect. A 356, 167 (1995).

[18] NKT Photonics, SuperK EXTREME supercontinuum lasers, http://www.nktphotonics.com/lasers-fibers/en/ product/superk-extreme-supercontinuum-lasers/.

[19] R. H. Fowler, The Analysis of Photoelectric Sensitivity Curves for Clean Metals at Various Temperatures, Phys. Rev. 38, 45 (1931).

[20] V. Ptitsyn (on behalf of the eRHIC Collaboration), eRHIC-Future electron-ion collider at BNL, AIP Conf. Proc. 842, 1046 (2006). 